\title{
Ewing Sarcoma of Larynx: A Rare Case in a 5-Year-Old Boy
}

\author{
Hamdan Ahmed Pasha (1), Shayan Khalid Ghaloo (D), Muhammad Wasif (1D, Moghira Iqbaluddin \\ Case Report $>$ Siddiqui (i), Nasir Ud Din \\ Department of Otorhinolaryngology-Head and Neck Surgery, Aga Khan University Hospital Karachi, Karachi, Pakistan
}

Abstract Ewing Sarcoma of the head and neck region is an extremely rare entity. Treatment usually involves surgery, chemotherapy and radiotherapy in varying sequences. We present the third case to date of Ewing sarcoma of the larynx in a paediatric population. A 5-year-old boy presented to emergency room with acute respiratory distress. Computerized tomography scan showed a mass in the supraglottis; he was intubated using vid- eolaryngoscope and tracheostomy was avoided, mass was removed by cold dissection. Final histopathologic examination revealed Ewing sarcoma. Further workup showed no systemic metastasis. Patient was advised adjuvant therapy which the family refused. Currently he is doing fine on 2 years of follow-up.

Keywords: Ewing sarcoma, larynx, paediatric, surgery
ORCID IDs of the authors: H.A.P. 0000-0002-6620-7544; S.K.G. 0000-0002-3237-0669; M.W. 0000-0002-3760-484x; M.I.S. 0000-0001-9169-5802; N.U.D. $0000-0002-1142-4218$

Cite this article as: Pasha HA, Ghaloo SK, Wasif M, Siddiqui MI, Din NU. Ewing Sarcoma of Larynx: A Rare Case in a 5-Year-0ld Boy.

Turk Arch Otorhinolaryngol 2020; 58(1): 65-8.

\section{Corresponding Author:}

MuhammadWasif; wasif_siddiq313@yahoo.com

Received Date: 06.10.2019

Accepted Date: 26.12 .2019

Available Online Date: 26.03 .2020

Content of this journal is licensed under a Creative Commons Attribution 4.0 International License. Available online at www.turkarchotolaryngol.net

\section{Introduction}

Children presenting to pediatric emergency with stridor is not uncommon. Most common causes of stridor in pediatric population are laryngomalacia, subglottic stenosis or vocal cord paralysis (1). We report the case of a 5-year-old boy who presented with stridor to the emergency room and was found to have a laryngeal mass that was excised and diagnosed as Ewing sarcoma. The clinical presentation, management and outcome has been discussed with a review of the literature. Only two pediatric cases of Ewing sarcoma have been previously reported more than two to three decades ago (2). Overall, a total of seven cases with Ewing sarcoma have been reported to occur in the larynx.

\section{Case Presentation}

A 5-year-old boy presented to the emergency department with progressive change in his voice for eight months, difficulty in breathing for six months and stridor for three days. He had no difficulty in swallowing. The mother of the child also denied any history of foreign body ingestion, intubation, trauma or fever. On examination, the child was anxious, restless and in moderate respiratory dis- tress. Nebulization with epinephrine and steroid injection improved the condition of the child with reduced work of breathing and resulted in a better respiratory rate. The child was then moved for a computerized tomography (CT) scan that showed a rounded soft tissue density mass of $18 \times 16 \mathrm{~mm}$ in the supraglottic region and the ventricles, it was abutting the epiglottis, likely representing papilloma (Figure 1).

The child was moved to the operating room. He was examined with the help of a videolaryngoscope, and a mass of about $2 \times 2 \mathrm{~cm}$ was seen to obstruct the upper airway (Figure 2). He was intubated and tracheotomy was avoided. The tumor was firm in consistency and was excised in piecemeal with cold knife technique (Figure 3). The patient was extubated uneventfully, with no stridor or dyspnea. He was observed for 24 hours and discharged in stable condition on the second post-operative day. Histopathological examination revealed squamous mucosa covered fragments showing infiltration by a neoplastic lesion arranged in diffuse sheets. The tumor cells were uniform, round, and exhibited hyperchromatic nu- 

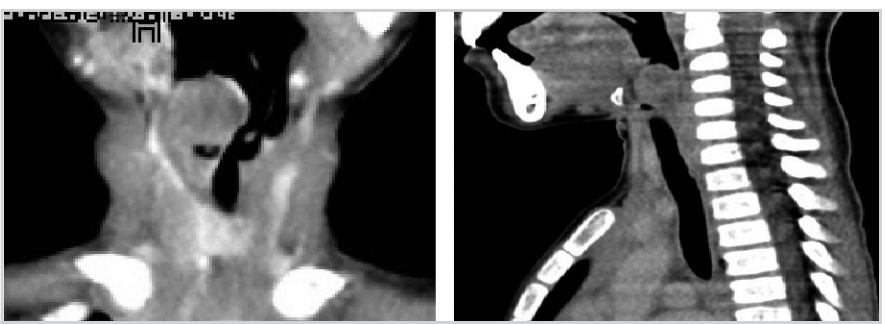

Figure 1. CT scan (coronal and sagittal views) showing mass in supraglottis

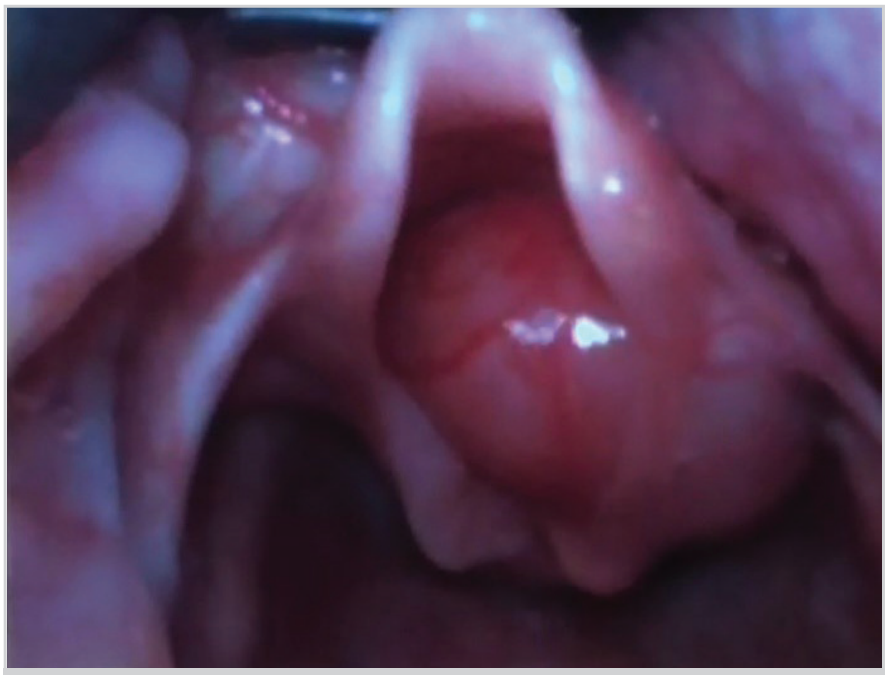

Figure 2. Video laryngoscopy showing laryngeal mass

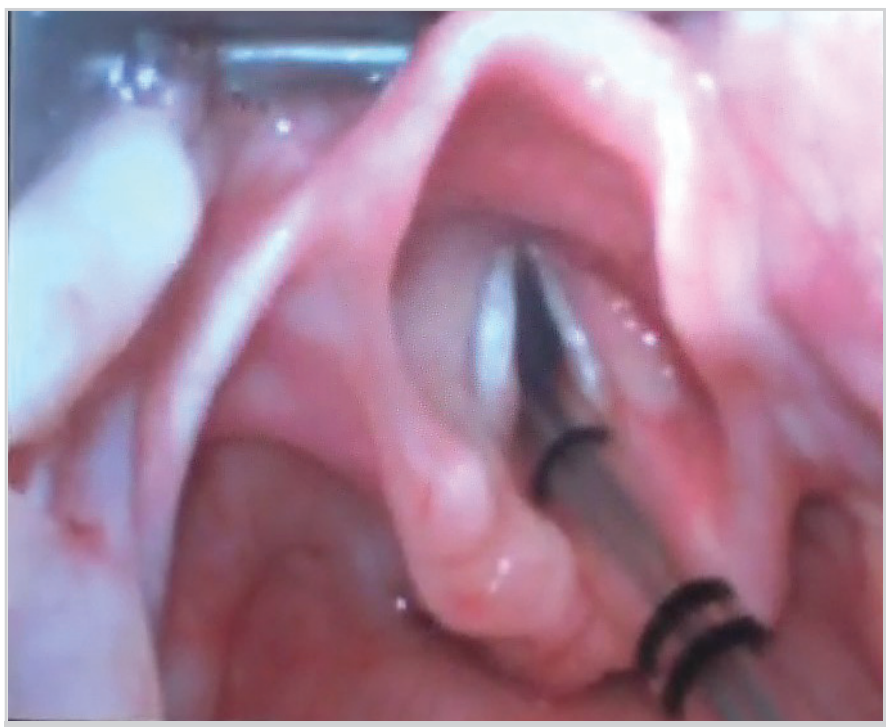

Figure 3. Adequate airway post excision of the laryngeal mass

clei with scant eosinophilic cytoplasm (Figures $4 \mathrm{a}-\mathrm{d}$ ). Increased mitotic figures were seen. Histologically, differential diagnoses of embryonal rhabdomyosarcoma, acute lymphoblastic lymphoma/leukemia and Ewing sarcoma were considered. A Periodic acid-Schiff (PAS) stain highlighted abundant cytoplasmic glycogen granules (Figure 5a). Immunohistochemical staining with CD99 was strongly membranous positive (Figure $5 \mathrm{~b}$ ) and cyclin D1 showed strong nuclear positivity. Desmin, leukocyte common antigen (LCA) and terminal deoxynucleotidyl transferase

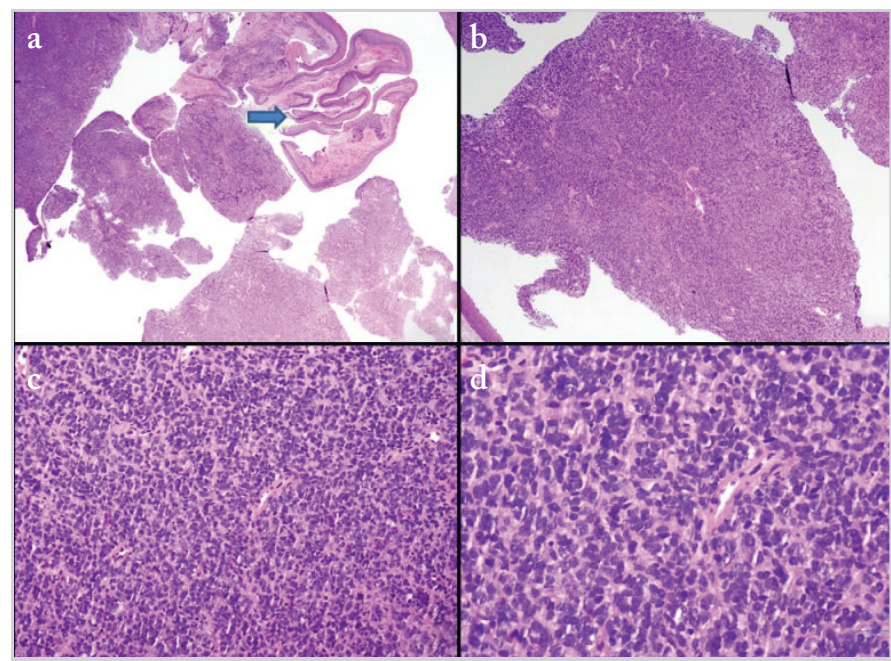

Figure 4. a-d. Low and high power examination of laryngeal Ewing sarcoma. Arrow in Figure a shows overlying stratified squamous epithelial lining. The tumor is arranged in diffuse sheets and has uniform round cells with hyperchromatic nuclei (H\&E stain)

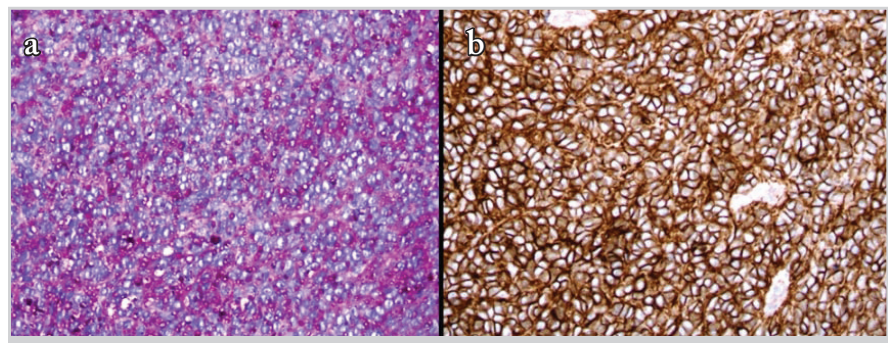

Figure 5. a, b. Abundant cytoplasmic granules are seen on PAS stain (a) and tumors cells showed strong membranous staining for CD99 immunohistochemical stain (b)

(TdT) were negative, thus ruling out rhabdomyosarcoma and acute lymphoblastic lymphoma, respectively. Cytokeratin AE1/ $\mathrm{AE} 3$ and p63 were negative, ruling out an undifferentiated carcinoma. CD117 was performed to rule out myeloid sarcoma and was negative. Histological features favored Ewing sarcoma. Fluorescence in situ hybridization (FISH) for Ewing sarcoma break apart (EWSR1) probe was performed and revealed nuclei in which one pair of the probe signals was split apart due to a rearrangement in the EWSR1 gene (green arrows, Figure 6). The FISH result confirmed the diagnosis of Ewing sarcoma. For further management, the patient was referred to paediatric oncology. His metastatic workup was done with bone scan and bone marrow biopsy. The results were negative with no evidence of spread of the disease. The patient was advised adjuvant chemotherapy with radiation, but the parents refused. He is under regular follow-up and at two years of follow-up has shown no symptoms or signs of recurrence. Informed consent was taken from the father to report this case.

\section{Discussion}

Ewing sarcoma is the second most frequent primary bone malignancy after osteosarcoma, mostly occurring in the flat bones of the axial skeleton and in the long bones. It has been divided into classic Ewing sarcoma of the bone, extraskeletal Ewing sarcoma (EES), and malignant peripheral primitive neuroectodermal tu- 


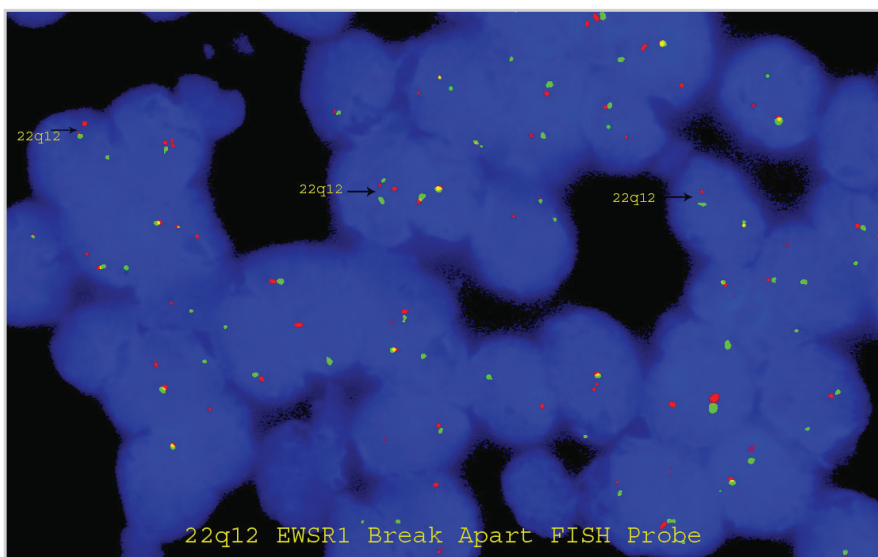

Figure 6. LSI EWSR1 Dual Color, Break Apart Rearrangement Probe revealed split apart green and red signals (black arrows) confirming the diagnosis of Ewing sarcoma along with morphology and immunoprofile

mor (pNET) (8). The debate on the exact cell of origin of Ewing sarcomas is yet unresolved. Histological features suggest a poorly differentiated tumor having both mesenchymal and neuroectodermal features. Head and neck Ewing sarcoma represents less than $9 \%$ of all Ewing sarcomas, with mandibular tumors being the most common (9). In a recent 43-year review from the Mayo Clinic only 17 patients had Ewing sarcoma in the head and neck region; of these most were found in bony structures, namely in the cervical spine and the skull (10). Majority of their patients were treated with surgery with or without adjuvant chemoradiation in varying sequences. They reported no case arising primarily in the larynx. Majority of the patients were adults and only one patient was under five years of age. Ellis et al. (11) used the Surveillance, Epidemiology, and End Results (SEER) database to publish the largest series of Ewing sarcoma in the head and neck. They found Ewing sarcomas in the head and neck to be smaller, with lower rates of metastasis at presentation when compared to those originating at other sites; but did not deem it to be a separate clinical entity, and the prognosis of Ewing sarcoma in the head and neck was not different than that at other primary sites. The presence or absence of metastasis is a key prognostic factor for Ewing sarcoma. In the head and neck Ewing sarcomas, the frequency of metastasis at presentation is lower, although larger tumor volumes are associated with worse survival rates (12-14).

The youngest pediatric patient of laryngeal Ewing sarcoma was a 1-day-old male baby who presented with respiratory distress and stridor at the time of birth. Laryngoscopy and bronchoscopy showed a right sided solitary mass causing obstruction of the glottis. A biopsy was taken for frozen section which revealed an undifferentiated malignant tumor. His metastatic workup was negative. A tracheostomy was performed as the tumor was obstructing the airway. A total laryngectomy was performed on the $10^{\text {th }}$ day of life. Final diagnosis was a malignant undifferentiated sarcoma consistent with an extraskeletal Ewing sarcoma. Patient was alive and free of disease 2 years after the surgery (1). The second pediatric case was a 9-month-old male baby. A laryngectomy was done and patient received chemotherapy as well. The tumor recurred locally (2).
The other five cases of laryngeal Ewing sarcoma occurred in adults with age ranging from 33 to 74 years (mean 54.6 years; median 53 years) (3-7). Three were male and two were female. Yang and Hong (3) reported laryngeal Ewing sarcoma in a 74-year-old male. The patient presented with acute respiratory distress; after emergency tracheostomy, workup revealed Ewing sarcoma. He underwent laryngectomy, neck dissection and adjuvant radiation. The patient stayed disease-free for six months.

Another 68-year-old male was reported with a 3-month history of hoarseness and aphonia. He had neoplastic infiltration occupying the anterior commissure, both vocal cords and the laryngeal ventricles. The patient refused laryngectomy and underwent induction chemotherapy followed by radiation. Complete regression of the tumor was seen and patient did well at 30 months follow-up (4).

The current treatment line for Ewing sarcoma includes six cycles of neoadjuvant chemotherapy, followed by local therapy, i.e., surgery, radiotherapy or both, with continued chemotherapy for a year. The initial evaluation before treatment should include imaging which can assess the degree of compromised bone cortex and its relation to adjacent anatomic landmarks. Magnetic resonance imaging remains the gold standard for radiographic characterization of Ewing sarcoma as it can determine the relationship of the tumor with the adjacent structures. Patients should also receive chest $\mathrm{CT}$ and technetium $\mathrm{Tc} 99 \mathrm{~m}$ bone scanning or PET scanning in order to identify metastatic lesions.

\section{Conclusion}

Ewing sarcoma of the larynx is a rare entity especially in the paediatric age group. The diagnostic challenge lies in differentiating this tumor from other causes of round blue cell tumors. Extensive immunostaining and FISH confirm the diagnosis. Treatment protocols are not uniform owing to the rare occurrence. Our patient was managed by surgery alone and no recurrence was seen on two years follow-up.

Informed Consent: Written informed consent was obtained from the parents of the patient who participated in this case.

Peer-review: Externally peer-reviewed.

Author Contributions: Concept - H.A.P.; Design - S.K.G., M.I.S.; Literature Search - M.W.; Writing - H.A.P., S.K.G., M.W., M.I.S., N.U.D.; Critical Reviews - N.U.D.

Conflict of Interest: The authors have no conflicts of interest to declare.

Financial Disclosure: The authors declared that this study has received no financial support.

\section{References}

1. Nolder AR, Richter GT. The infant with noisy breathing. Curr Treat Options Pediatr. 20151; 1: 224-33. [Crossref]

2. Abramowsky CR, Witt WJ. Sarcoma of the larynx in a newborn. Cancer 1983; 51: 1726-30. 
3. Jones JE, McGill T. Peripheral primitive neuroectodermal tumors of the head and neck. Arch Otolaryngol Head Neck Surg 1995; 121: 1392-5. [Crossref]

4. Yang YS, Hong KH. Extraskeletal Ewing's sarcoma of the larynx. J Laryngol Otol 2004; 118: 62-4. [Crossref]

5. Wygoda A, Rutlowski T, Ponikiewska D, Hejduk B, Skladowski K. Ewing's sarcoma of the larynx. Effective treatment with organ preservation. Strahlenther Onkol 2013; 189: 586-9. [Crossref]

6. Lynch MC, Baker A, Drabick JJ, Williams N, Goldenberg D. Extraskeletal Ewing's sarcoma arising in the larynx. Head Neck Pathol 2014; 8: 225-8. [Crossref]

7. Ijichi K, Tsuzuki T, Adachi M, Murakami S. A peripheral primitive neuroectodermal tumor in the larynx: a case report and literature review. Oncol Lett 2016; 11: 1120-4. [Crossref]

8. Maroun CA, Khalifeh I, Tfayli A, Moukarbel RV. Primary Ewing sarcoma of the larynx with distant metastasis: a case report and review of the literature. Curr Oncol 2019; 26: e574-7. [Crossref]

9. Windfuhr JP. Primitive neuroectodermal tumor of the head and neck: incidence, diagnosis, and management. Ann Otol Rhinol Laryngol 2004; 113: 533-43. [Crossref]
10. Vaccani JP, Forte V, de Jong AL, Taylor G. Ewing's sarcoma of the head and neck in children. Int J Pediatr Otorhinolaryngol 1999; 48: 209-16. [Crossref]

11. Olson MD, Van Abel KM, Wehrs RN, Garcia JJ, Moore EJ. Ewing sarcoma of the head and neck: The Mayo Clinic experience. Head Neck 2018; 40: 1999-2006. [Crossref]

12. Ellis MA, Gerry DR, Neskey DM, Lentsch EJ. Ewing sarcoma of the head and neck: analysis of 183 cases with comparison to non-head and neck sites. Ann Otol Rhinol Laryngol 2017; 126: 179-84. [Crossref]

13. Duchman KR, Gao Y, Miller BJ. Prognostic factors for survival in patients with Ewing's sarcoma using the surveillance, epidemiology, and end results (SEER) program database. Cancer Epidemiol 2015; 39: 189-95. [Crossref]

14. Bakhshi S, Pathania S, Mohanti BK, Thulkar S, Thakar A. Therapy and outcome of primitive neuroectoderm al tumor of the jaw. Pediatr Blood Cancer 2011; 56: 477-81. [Crossref]

15. Bacci G, Longhi A, Ferrari S, Mercuri M, Versari M, Bertoni F. Prognostic factors in non-metastatic Ewing's sarcoma tumor of bone: an analysis of 579 patients treated at a single institution with adjuvant or neoadjuvant chemotherapy between 1972 and 1998. Acta Oncol 2006; 45: 469-75. [Crossref] 